\title{
Simulación de sistemas eléctricos con cargas no lineales y variantes en el tiempo
}

\author{
Simulation of electric systems with non-linear and time-variant loads
}

\author{
William Carvajal Carreño ${ }^{1} \quad$ Gabriel Ordóñez Plata ${ }^{1} \quad$ Andrés L. Moreno Wandurraga $^{2}$ \\ César A. Duarte Gualdrón ${ }^{1}$
}

Recibido 9 de noviembre de 2009, aceptado 17 de diciembre de 2010

Received: November 9, 2009 Accepted: December 17, 2010

\begin{abstract}
RESUMEN
En este artículo se propone una metodología, implementada en un programa de computador por medio de una interfase gráfica con el usuario (GUI), que permite simular redes eléctricas con elementos no lineales (principalmente convertidores de potencia), tanto en el dominio del tiempo como en el dominio de la frecuencia. El método propuesto combina dos tipos de análisis en una herramienta flexible, de forma que se puedan aprovechar las ventajas de cada uno de ellos, y que permita analizar sistemas lineales y no lineales en el dominio del tiempo, sistemas lineales en el dominio de la frecuencia y sistemas mixtos utilizando este método híbrido. La herramienta es un instrumento de cálculo versátil y completo que puede ser usado de una manera sencilla, para los estudios de análisis de propagación de armónicos en las redes eléctricas. Se presentan ejemplos extraídos de la bibliografía utilizada en cursos de sistemas de potencia, circuitos eléctricos y electrónica de potencia, así como sistemas de prueba para análisis de cargas no lineales o variantes en el tiempo, generados como marco de referencia para las simulaciones de armónicos. Se analizan los resultados y se hacen observaciones acerca de las características de convergencia y estabilidad algorítmica del método propuesto. Dada la complejidad y carga computacional del análisis del método híbrido propuesto, se han seleccionado modelos de elementos, modelos de los componentes del sistema y algoritmos apropiados, para que la solución del problema se realice dentro de límites razonables de tiempo de simulación y consumo de recursos del equipo de cómputo.
\end{abstract}

Palabras clave: Armónicos, tiempo, frecuencia, híbrido, iterativo, lineal, Norton, SASP.

\begin{abstract}
This paper deals with a methodology implemented in a computer program with a Graphic User Interface (GUI) that allows us to simulate electrical networks (mainly power converters) with non linear elements. The proposed approach mixes two analysis types in a flexible tool, in that way, we can take advantage of the features of each of them and it permits to analyze linear and non linear systems in the time domain, linear systems in the frequency domain and mixed systems using the proposed hybrid method. The tool is intended to be a versatile and comprehensive computation instrument. Besides, it can be used in a straightforward way to analyze harmonic propagation in electrical networks. Examples from the literature used in electrical circuits, power systems, and power electronics courses are shown. Likewise, in order to analyze non-linear or time-varying loads, test systems were created as a benchmark for testing simulators of harmonics in electrical systems. Simulation results are analyzed and observations about the convergence features and algorithm stability of the proposed method are given. Due to the complexity and computational burden of hybrid analysis, we have selected element models, system models and suitable algorithms to guarantee that the solution is reasonable in terms of simulation time and computer resources.
\end{abstract}

Keywords: Harmonics, time, frequency, hybrid, iterative, linear, Norton, SASP.

1 Escuela de Ingeniería Eléctrica, Electrónica y Telecomunicaciones. Universidad Industrial de Santander. Carrera 27, calle 9, Ciudad Universitaria. Bucaramanga, Colombia.E-mail: carvajal_william@hotmail.com; gaby@uis.edu.co; cedagua@uis.edu.co

2 Coordinación de personal recursos y planeación. Corriente Alterna Ltda. Bogotá, Colombia. E-mail: amorenow@ gmail.com 


\section{INTRODUCCIÓN}

Los sistemas eléctricos de corriente alterna son diseñados para operar con tensiones y corrientes sinusoidales. Sin embargo, las cargas no lineales y/o variantes con el tiempo distorsionan las formas de onda de tensión y corriente en estado estable provocando la aparición de las componentes armónicas. El análisis de la generación y propagación de tales componentes a través del sistema eléctrico se denomina flujo de potencia armónica [1].

Los estudios de armónicos son utilizados para cuantificar la distorsión de las ondas de tensión y corriente en varios puntos en un sistema eléctrico y determinar si existen condiciones resonantes que deban ser mitigadas [2].

La investigación se ha centrado en la evaluación del flujo de potencia armónico en las redes eléctricas; esto condujo a la disponibilidad de una gran variedad de programas de computador en donde se implementan técnicas de formulación y solución de los problemas de propagación armónica [3-6].

El acoplamiento entre las componentes armónicas es una relación no lineal, la linealización alrededor de un punto de operación solo será valida en la vecindad de ese punto. El cálculo bajo estas condiciones se hace difícil y debe tenerse una actualización iterativa de resultados para obtener valores dentro de un rango de error admisible. La carga computacional se aumenta en proporción directa al tamaño del sistema y el número de componentes armónicas [8-9].

La eficiencia computacional en esta evaluación puede ser mejorada si se reemplazan los cálculos en el dominio de la frecuencia para los elementos no lineales por operaciones en el dominio del tiempo seguidas por transformadas de Fourier; de esta manera se desacoplan las componentes armónicas. El acople de componentes armónicas puede conseguirse mediante un proceso iterativo [10-13].

\section{CONSIDERACIONES GENERALES}

La obtención de modelos de cargas electrónicas no lineales puede dividirse en dos categorías básicas, dependiendo de los objetivos del estudio. La primera cubre todas las evaluaciones de estado estable. El objetivo es obtener la respuesta del sistema a las componentes armónicas desde los subsistemas no lineales. En este tipo de estudios la inyección de corrientes armónicas puede asumirse independiente de las variaciones de tensión en el punto de acople común (PCC), y el modelo del subsistema no lineal puede ser reducido a un circuito equivalente en paralelo [1, 14].

El segundo tipo de análisis abarca un rango más extenso y complejo de problemas prácticos. En muchas aplicaciones, la operación de un subsistema depende estrechamente del estado de operación del sistema conectado. Algunos ejemplos de este tipo de aplicaciones son: un sistema de compensación estática de VAR, filtros activos o variadores de velocidad.

Debido a que en estos casos el subsistema electrónico de potencia afecta directamente la operación total del sistema, es inaceptable un tratamiento por separado del sistema de alimentación y el sistema de electrónica de potencia [15-16].

\section{LINEAMIENTOS GENERALES}

En la simulación de circuitos y sistemas debe existir un equilibrio entre la complejidad de los modelos y el tiempo de simulación necesario para obtener resultados dentro de una incertidumbre especificada.

\section{Representación de sistemas de electrónica de potencia}

Generalmente, no se representan todos los elementos; lo que debe simularse es la característica en terminales del subsistema. De esta forma puede sugerirse el siguiente procedimiento para reducir la complejidad del modelo [17].

1. Utilizar elementos equivalentes para combinaciones serie y paralelo.

2. Utilizar el modelo más simple para cada aplicación.

3. Representar sólo las características en terminales del elemento.

4. Utilizar modelos modulares para sistemas a gran escala.

\section{Errores de simulación}

Los errores de simulación pueden provenir de la aproximación de los modelos del sistema, elementos de circuito adicionados para control de oscilación 
numérica [18], truncamiento relacionado con el paso de integración, estructura del programa y métodos de solución o condiciones iniciales incorrectas.

El paso de integración recomendado no debe superar $1 / 5$ a 1/20 del periodo de la frecuencia más alta de interés. Para reducir los errores por condiciones iniciales se permite al programa ejecutar un periodo de tiempo para alcanzar una condición inicial corregida [19].

Para simulaciones en corriente continua el tiempo total de simulación debe ser mayor a tres veces la constante de tiempo $(\tau)$ mas grande. En sistemas de corriente alterna se deben simular varios ciclos para permitir la estabilización de la forma de onda.

\section{MODELOS DE LOS COMPONENTES DEL SISTEMA}

Los componentes del sistema pueden ser elementos de características lineal o no lineal. A continuación se describen los modelos de los principales elementos y subsistemas utilizados.

\section{Modelo de la red lineal}

El modelo de una red lineal trifásica se realiza en el dominio de la frecuencia y por fase para permitir el tratamiento de circuitos desequilibrados.

\section{Modelo de las cargas}

Las cargas se pueden representar en forma agregada en un punto determinado del sistema [7]. La carga se modela con una conductancia $G$ en paralelo con una susceptancia $B$, a partir de la potencia activa $(P)$ y reactiva $(Q)$ consumidas en cada fase, y la tensión nominal $(V)$ a frecuencia fundamental.

\section{Líneas de transmisión}

Una línea de transmisión se compone de resistencia, inductancia y capacitancia distribuidas en su longitud. Una línea equilibrada de longitud media puede ser modelada mediante su equivalente $\pi$ (Figura 1).

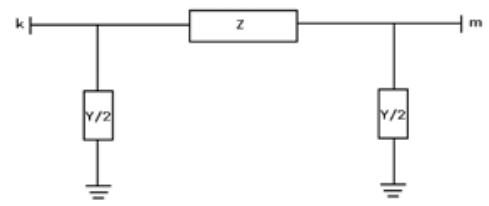

Figura 1. Equivalente $\pi$ de una línea de transmisión

\section{Transformadores}

La matriz de admitancias de nodo de los transformadores se forma a partir de las matrices de admitancia de ramas obtenidas de cada devanado y las de la admitancia de la rama de magnetización. La matriz que relaciona las tensiones (Vrama) y corrientes (Irama) en los devanados del transformador es la matriz de admitancia de devanados, definida por:

$$
\text { Irama }=\text { Yrama } * \text { Vrama }
$$

A partir de esta matriz se obtiene la matriz de admitancias nodales por medio de una matriz $(C)$ que la relaciona al grupo de conexión del transformador de la siguiente manera:

$$
\begin{aligned}
& \text { Vrama }=C * \text { Vnodo } \\
& \text { Inodo }=C^{t} * \text { Irama }
\end{aligned}
$$

Sustituyendo se obtiene:

$$
\text { Ynodo }=C^{t} * \text { Yrama } * C
$$

La matriz Yrama se puede ordenar en submatrices que representan los acoplamientos primarios, secundarios y mutuos, como se muestra en la ecuación (5).

$$
\left[\begin{array}{c}
I_{p} \\
I_{s}
\end{array}\right]=\left[\begin{array}{cc}
Y_{p p} & Y_{p s} \\
Y_{s p} & Y_{s s}
\end{array}\right]\left[\begin{array}{c}
V_{p} \\
V_{s}
\end{array}\right]
$$

\section{Fuentes de tensión y corriente}

Debido a que se trabaja con equivalentes Norton, y para evitar singularidades en la matriz de impedancias, todas las fuentes de tensión se consideran en serie con una impedancia [20]. Ver Figura 2.

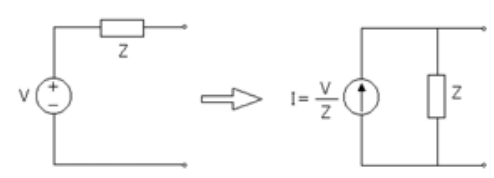

Figura 2. Equivalente Norton de una fuente de tensión.

\section{Filtros pasivos}

Estos filtros, en serie o paralelo, atenúan las componentes armónicas y compensan el factor de potencia a la frecuencia fundamental. Las principales configuraciones de filtros pasivos se muestran en la Figura 3. 


\section{Cargas no lineales}

En las redes eléctricas los sistemas no lineales generalmente están representados por los semiconductores de potencia y la saturación de los núcleos de los transformadores [21].

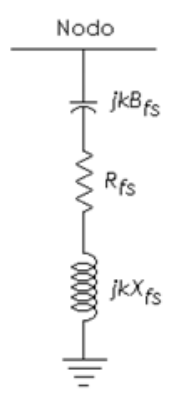

Filtro sintonizado

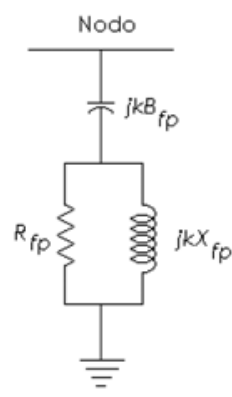

Filtro pasabajo
Figura 3. Filtros pasivos para sistemas de distribución.

\section{Semiconductores}

Los elementos semiconductores pueden ser analizados con una conductancia no lineal dada por la relación entre tensión $(u)$ e intensidad $(i)$ descrita por (6):

$$
i=g_{n}(u)
$$

Si esta conductancia está conectada a un circuito resistivo lineal, cuyo equivalente Norton en bornes de la resistencia es:

$$
i=i_{N}-g \cdot u
$$

La obtención de la tensión y la corriente será el resultado de la solución de la ecuación no lineal (8)

$$
g_{n}(u)=i_{N}-g . u
$$

La solución de esta ecuación se obtiene por medio de un método iterativo, al hallar los valores de $i_{o}$ y $u_{o}$.

En un circuito con fuentes de corriente alterna, el valor del equivalente Norton en bornes de la resistencia no lineal cambia en cada instante de tiempo, debido a esto se tiene que obtener el circuito discreto asociado cada vez que se avance en un paso de la simulación. El proceso iterativo para calcular el equivalente Norton se debe actualizar en cada instante de tiempo.

Al trabajar con circuitos linealizados por tramos, se hace el proceso más eficiente y práctico [2223]. En este caso, las resistencias o conductancias tendrán valores distintos en cada tramo $m$, que se define por:

- Las intensidades límite que definen la extensión del tramo: $i_{m-1}$ e $i_{m}$

- La tensión de una de las intensidades límite: $u_{m-1}$

- La conductancia de cada tramo $g_{m}$

Con estos valores se obtiene el equivalente Norton del tramo $m$, cuando la corriente de la resistencia no lineal está entre $i_{m-l}$ e $i_{m}$. Las ecuaciones son (9):

$$
\begin{aligned}
& i\left(t_{n}\right)=g_{m} u\left(t_{n}\right)+i^{\prime}{ }_{m} \\
& i_{m}{ }_{m}=i_{m-1}-g_{m} u_{m-1}
\end{aligned}
$$

El equivalente lineal a tramos es como en la Figura 4.
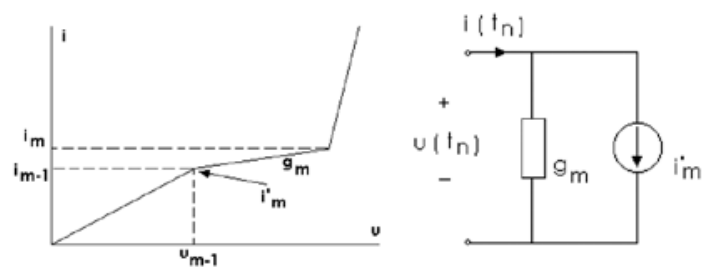

Figura 4. Circuito lineal por tramos y su equivalente Norton.

El procedimiento a seguir es el siguiente:

- Para un tramo dado, se incluyen los parámetros del equivalente Norton en la matriz de admitancias del sistema y en el vector de corrientes, obteniéndose así las tensiones y corrientes en el elemento.

- Se comprueba si estas magnitudes están dentro del tramo elegido. Si no, se analiza el equivalente Norton del tramo en el que esté comprendida la corriente obtenida y se regresa al paso anterior.

Los semiconductores, modelados como interruptores, son resistencias no lineales con dos posibles estados, baja impedancia o alta impedancia. La característica lineal por tramos de un diodo es:

$$
\begin{gathered}
i=g_{m} \cdot u \text { para } i>0 \\
i=0 \text { para } i<0
\end{gathered}
$$

Donde $g_{m}$ es una conductancia de valor muy alto, idealmente infinito. Esto se observa en la Figura 5. 
La característica tensión-corriente de los tiristores es determinada por el disparo de compuerta, que hace que el elemento pase del estado de bloqueo al de conducción si se encuentra polarizado directamente. Este disparo ocurre un tiempo después del cruce por cero con pendiente positiva de la tensión del elemento.

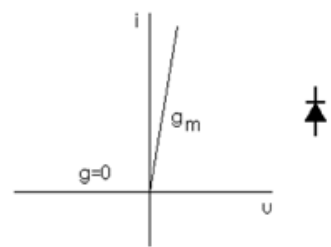

Figura 5. Característica tensión-corriente de un semiconductor.

En el modelo del tiristor, a diferencia del diodo, se consideran la tensión de control y el ángulo de disparo. La tensión de control es idealmente entre ánodo y cátodo, pero generalmente se toma un valor accesible para los elementos de control y una forma de onda con una distorsión armónica total (THD) baja. En este trabajo se considera como tensión de control el valor de una señal sinusoidal pura.

Los transistores de potencia, MOSFETs e IGBTs son interruptores en estado de bloqueo o conducción dependiendo de la polaridad de la tensión en sus terminales y la señal de compuerta o base, y requieren de una señal externa permanente en la base o compuerta para que permanezca en conducción.

\section{MÉTODOS DE ANÁLISIS DE LOS SISTEMAS ELÉCTRICOS}

\section{Método híbrido tiempo-frecuencia}

El análisis de redes compuestas de elementos lineales y no lineales, analizados en los dominios del tiempo y la frecuencia, exige el análisis en cada uno de los dominios de las subredes que conforman el sistema. El paso siguiente consiste en establecer cómo relacionar los resultados de cada dominio. $\mathrm{La}$ esencia del método híbrido radica en esta técnica [10, 12, 24-25].

\section{Equivalente de la red lineal}

Luego de haber obtenido la matriz de admitancias de nodo de la red lineal, esta red se reduce al equivalente en bornes de cada uno de los elementos no lineales.
Este equivalente se obtiene separando las tensiones y las corrientes de los nodos donde existen elementos no lineales $\left(I_{N L}, U_{N L}\right)$ de los que solamente tienen cargas lineales $\left(I_{L}, U_{L}\right)$, dividiendo la matriz de admitancias en cuatro submatrices (11).

$$
\left[\begin{array}{c}
I_{L} \\
\hline I_{N L}
\end{array}\right]=\left[\begin{array}{l|l}
Y_{L L} & Y_{L N} \\
\hline Y_{N L} & Y_{N N}
\end{array}\right]\left[\begin{array}{c}
U_{L} \\
\hline U_{N L}
\end{array}\right]
$$

Eliminando el término $U_{L}$ se obtiene:

$$
\begin{gathered}
I_{N L}=\left[Y_{N N}-Y_{N L} Y_{L L}^{-1} Y_{L N}\right] U_{N L}+Y_{N L} Y_{L L}^{-1} I_{L} \\
Y_{E Q}=Y_{N N}-Y_{N L} Y_{L L}^{-1} Y_{L N}
\end{gathered}
$$

Esta ecuación representa el equivalente Norton de la red referido a los nodos en los que hay conectados elementos no lineales, la cual puede expresarse como:

$$
I_{N L}+I_{E Q}=Y_{E Q} U_{N L}
$$

\section{Inicialización del proceso iterativo}

Las tensiones iniciales se obtienen resolviendo un flujo de cargas en la red lineal a frecuencia fundamental $(60 \mathrm{~Hz})$. Los circuitos no lineales se consideran como cargas lineales cuyo consumo es un dato de entrada al algoritmo [26].

Con las tensiones iniciales se obtiene un vector de inyecciones de corriente que representa el equivalente Norton de la red lineal de la siguiente forma:

$$
I_{E Q}=Y_{E Q} U_{N L O}
$$

\section{Flujo de carga armónico}

Este flujo permite calcular la tensión en las barras, para cada frecuencia. Si las cargas no lineales tienen una componente armónica a la frecuencia $h$, se tiene:

$$
\left[V^{h}\right]\left[Y_{\text {Barra }}^{h}\right]=\left[I^{h}\right]
$$

Donde la matriz de admitancias de barra se obtiene por inspección. Por lo tanto, se puede calcular el flujo de carga armónico en cada barra del sistema de la siguiente forma: 


$$
\left[V^{h}\right]=\left[I^{h}\right]\left[Z_{\text {Barra }}^{h}\right]
$$

\section{Resolución de la red en el dominio del tiempo}

En este trabajo se utiliza como algoritmo de integración la regla trapezoidal, y el método de Euler implícito para corregir sus oscilaciones numéricas.

\section{Método de análisis nodal con equivalentes Norton}

En este método se modela un circuito dinámico no lineal como uno resistivo lineal. Todos los elementos se modelan como un equivalente Norton resistivo cuyos valores cambian en cada iteración. Con los equivalentes Norton se forma la matriz de admitancias nodales y el vector de fuentes de corriente y se calcula el vector de tensiones nodales y a partir de él todas las demás magnitudes del circuito [13].

\section{Modelos de bobinas y condensadores}

Sea una bobina lineal definida por la ecuación (18):

$$
u=L \frac{d i}{d t}
$$

Al integrar (18) se obtiene

$$
\frac{1}{L} \int_{t_{n}}^{t_{n+1}} u d t=\int_{i\left(t_{n}\right)}^{i\left(t_{n+1}\right)} d i
$$

Considerando ahora la regla trapezoidal se tiene:

$$
\frac{h}{2 L}\left[u\left(t_{n+1}\right)+u\left(t_{n}\right)\right]=i\left(t_{n+1}\right)-i\left(t_{n}\right)
$$

Donde $h$ es el paso de integración. Reordenando:

$$
\begin{gathered}
i\left(t_{n+1}\right)=g_{b} u\left(t_{n+1}\right)+i_{b}^{n} \\
i_{b}^{n}=i\left(t_{n}\right)+g_{b} u\left(t_{n}\right) \\
g_{b}=\frac{h}{2 L}
\end{gathered}
$$

Estas ecuaciones se interpretan como una conductancia en paralelo con una fuente de corriente que varía en cada iteración, tomando el valor del instante (paso) anterior, como se ilustra en la Figura 6.

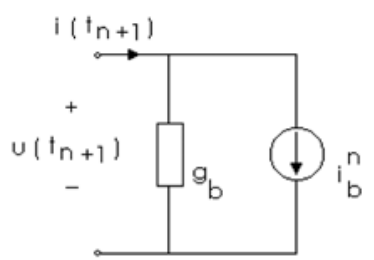

Figura 6. Circuito discreto para un elemento dinámico.

Para el condensador se realiza un procedimiento similar y se obtiene la siguiente ecuación:

$$
\begin{gathered}
i\left(t_{n+1}\right)=g_{c} u\left(t_{n+1}\right)+i_{c}^{n} \\
i_{c}^{n}=-i\left(t_{n}\right)-g_{c} u\left(t_{n}\right) \\
g_{c}=\frac{2 C}{h}
\end{gathered}
$$

Utilizando el algoritmo de Euler implícito las ecuaciones de la bobina son:

$$
\begin{aligned}
& i_{b}^{n}=i\left(t_{n}\right) \\
& g_{b}=\frac{h}{L}
\end{aligned}
$$

Mientras que las del condensador corresponden a:

$$
\begin{aligned}
& i_{c}^{n}=-g_{c} u\left(t_{n}\right) \\
& g_{c}=\frac{C}{h}
\end{aligned}
$$

Con las conductancias de rama y las fuentes de corriente se forma la matriz de admitancias y el vector de corrientes nodales utilizando la matriz de incidencias.

\section{Modelo de bobinas lineales acopladas}

Para un conjunto de bobinas acopladas conectadas entre los nodos i y k; como se muestra en la Figura 7 , la relación entre las tensiones, las corrientes, las inductancias propias y mutuas se puede expresar así:

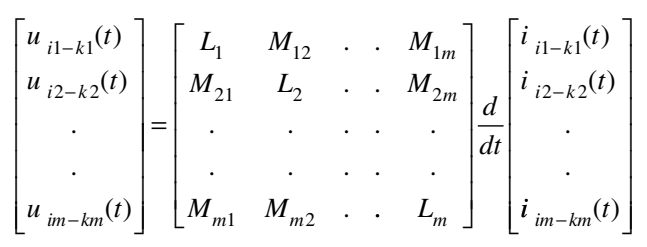




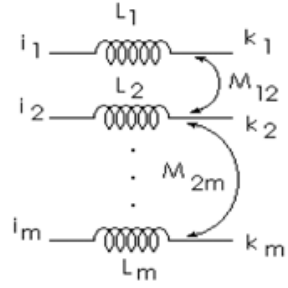

Figura 7. Esquema de bobinas acopladas.

La matriz de conductancias corresponde a la inversa de la matriz de inductancias propias y mutuas multiplicada por la mitad del paso de integración. El vector de fuentes de corriente corresponde al valor histórico de la corriente en el paso anterior $n$.

Las fuentes de corriente de los equivalentes Norton se añaden al vector de fuentes de corriente de rama y las conductancias se suman a la matriz de admitancias nodales.

\section{Solución de las ecuaciones nodales}

Luego de obtener los equivalentes Norton para cada rama en cada instante de tiempo, se forma la matriz de admitancias y el vector de corrientes nodales, así:

$$
\left[u_{n}\right]=\left[y_{n}\right]^{-1}\left[i_{n}\right]
$$

\section{Oscilaciones numéricas de la regla trapezoidal}

Al realizar la integración de las ecuaciones diferenciales en los elementos no lineales, la corriente varía bruscamente al pasar de un tramo a otro de su característica. Si es la corriente por una inductancia se producirán oscilaciones en su tensión. Afortunadamente, las conductancias de los equivalentes Norton para la regla trapezoidal con un paso de integración $h$ son equivalentes a las de Euler implícito para un paso $h / 2$. Debido a esta similitud se corregirán las oscilaciones de esta manera:

- Se detecta el cambio en el elemento lineal a tramos, se modifica la matriz de impedancias y se recalculan las corrientes y tensiones en ese instante.

- Se calculan las fuentes de los equivalentes Norton asociados según las siguientes ecuaciones:

$$
\begin{gathered}
i_{b}^{n}=i\left(t_{n}\right) \\
i_{c}^{n}=-g_{c} u\left(t_{n}\right)
\end{gathered}
$$

- Luego se integra durante dos semipasos con el método de Euler, calculando los valores para $\mathrm{t}_{\mathrm{n}+1}$.

- Se calculan nuevamente las fuentes de los equivalentes Norton utilizando la regla trapezoidal y se continúa hasta encontrar otro cambio.

Este procedimiento evita las oscilaciones y además mantiene la secuencia de puntos, lo cual es importante para el análisis de Fourier.

\section{Algoritmo en el dominio del tiempo}

La solución de problemas en el dominio del tiempo consiste en la integración directa durante un intervalo de tiempo, en el cual se considera que el transitorio se ha extinguido y se ha alcanzado el estado estacionario. La convergencia se logra dentro del tiempo de simulación, dependiendo de las constantes del circuito.

\section{Interacción entre la red lineal y las redes no lineales}

En una red trifásica con uno o más sistemas no lineales, estos sistemas toman de la red corrientes no sinusoidales. Bajo este esquema de funcionamiento las tensiones en los nodos están distorsionadas y es conveniente representarlas por medio de su espectro.

Este problema se presenta en la Figura 8. Cada sistema no lineal puede estar conectado a la red lineal mediante uno o varios nodos comunes, denominados nodos frontera, cuyo número, en general, podrá ser diferente del número de sistemas no lineales.

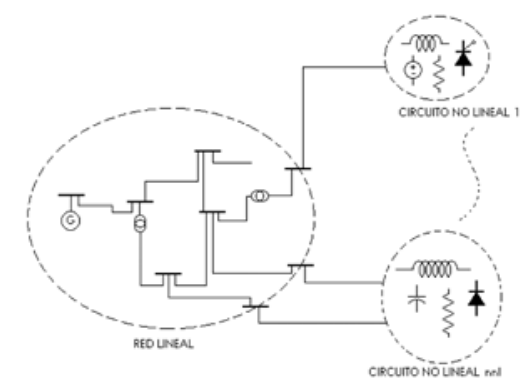

Figura 8. Representación del problema eléctrico. 
El procedimiento iterativo en los dominios del tiempo y de la frecuencia se presenta en la Figura 9.

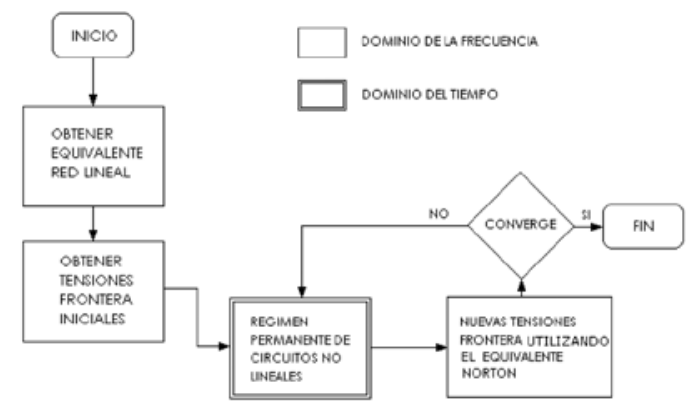

Figura 9. Solución híbrida al flujo de cargas armónico.

El método se descompone en los siguientes bloques fundamentales:

1. Formar el equivalente Norton de la red lineal en bornes de los nodos frontera.

2. Tomar un valor inicial de las tensiones frontera, que se obtiene de un flujo de cargas previo.

3. Con las tensiones de frontera se calculan las corrientes de los sistemas no lineales, calculando el estado estacionario en el dominio del tiempo.

4. Una vez obtenidas las corrientes frontera se calcula su espectro y se obtienen las nuevas tensiones frontera utilizando el equivalente Norton de la red lineal obtenido inicialmente.

5. Se repiten los puntos 3 y 4 hasta que se cumpla el criterio de convergencia.

Una vez modelada la red lineal en el dominio de la frecuencia y obtenido su equivalente Norton en bornes, se tiene el circuito de la Figura 10.

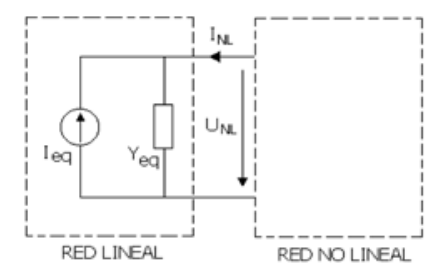

Figura 10. Representación del equivalente en bornes de cada carga no lineal.

Cada circuito no lineal $\boldsymbol{i}$ se considera como una función no lineal $f_{i}$ que relaciona la tensión en sus bornes $U_{N L, i}$ con la corriente que absorbe $I_{N L, i}$ es decir:

$$
I_{N L, i}=f_{i}\left(U_{N L}\right)
$$

El problema consiste en encontrar un conjunto de tensiones y corrientes que cumplan las condiciones impuestas por la red lineal y las redes no lineales, es decir, el sistema de ecuaciones formado por:

$$
\begin{aligned}
I_{N L}+I_{e q} & =Y_{e q} U_{N L} \\
I_{N L, 1} & =f_{1}\left(U_{N L}\right) \\
I_{N L, 2} & =f_{2}\left(U_{N L}\right) \\
\vdots & \\
I_{N L, n f} & =f_{n n f}\left(U_{N L}\right)
\end{aligned}
$$

Agrupando las ecuaciones de los circuitos no lineales en una ecuación en la que intervengan todas las corrientes y tensiones de los nodos frontera se tiene:

$$
\begin{aligned}
& I_{N L}=Y_{e q} U_{N L}-I_{e q} \\
& I_{N L}=f\left(U_{N L}\right)
\end{aligned}
$$

Igualando estas dos ecuaciones se obtiene que:

$$
\begin{aligned}
& f\left(U_{N L}\right)=Y_{e q} U_{N L}-I_{e q} \\
& f\left(U_{N L}\right)-Y_{e q} U_{N L}+I_{e q}=0
\end{aligned}
$$

Este problema se debe solucionar mediante un método iterativo. El método seleccionado para la solución de la ecuación es el método de punto fijo o Gauss [1].

El algoritmo de Gauss presenta la solución así:

$$
U_{N L}^{m+1}=Y_{e q}^{-1}\left[f\left(U_{N L}^{m}\right)+I_{e q}\right]
$$

Las corrientes de frontera se obtienen de la respuesta estacionaria de un circuito no lineal. Este problema se resuelve en tres pasos; primero se obtienen las tensiones que alimentan los circuitos no lineales, luego se obtiene la respuesta en estado estacionario de los circuitos no lineales y por último se evalúa el espectro de las corrientes frontera resultantes.

Se debe obtener la expresión en el dominio del tiempo de las tensiones frontera a partir de su espectro. 
Cada componente de frecuencia está representada por un fasor. Cada fasor de tensión en la red lineal representa la tensión entre fase y neutro del nodo. El valor de la fuente independiente de tensión del circuito no lineal correspondiente se obtiene pasando al dominio del tiempo y sumando la contribución de cada frecuencia de interés. Es decir, que para cada tensión frontera se tiene:

$$
u_{N L}(t)=\sqrt{2} \sum_{k}\left|U_{N L}\right| \cos \left(k w t+\theta_{U N L}\right)
$$

Con este valor en los circuitos no lineales se resuelve el problema de calcular el estado estacionario de todos los circuitos no lineales con las restricciones impuestas por la función de control de cada uno de ellos.

Una vez resueltos los problemas de régimen permanente en el dominio del tiempo, se obtienen las corrientes de los elementos no lineales $i_{N L}$. Luego se calcula el espectro de la corriente (dominio de la frecuencia) aplicando la transformada rápida de Fourier (FFT) de $N$ puntos en un periodo de la solución de estado estacionario:

$$
I_{N L}=F F T\left(i_{N L}\right)
$$

Estas nuevas corrientes se introducen en las ecuaciones de la red lineal para obtener las nuevas tensiones en bornes de los circuitos no lineales con la ecuación (33); este proceso se repite hasta que la diferencia sea menor que la tolerancia prefijada.

\section{HERRAMIENTA DE SIMULACIÓN SASP, SISTEMAS DE PRUEBA Y ANÁLISIS DE RESULTADOS}

La metodología planteada se ha validado utilizando un programa sobre la plataforma MATLAB 7 SP3. Este programa cuenta con interfase gráfica con el usuario (GUI) y con una estructura de inclusión de modelos, en un formato similar al del programa SPICE, permite gran flexibilidad para la creación de nuevos elementos y sistemas a partir del equivalente Norton básico.

Los sistemas de prueba han sido desarrollados por diferentes investigadores en el área y su propósito es brindar una herramienta de simulación, dada la dificultad de obtener medidas reales de sistemas [14].

\section{El Programa SASP}

La integración de los modelos de los componentes del sistema y los métodos en frecuencia, tiempo e híbrido, fue implementada en la interfase gráfica SASP (Simulación de Armónicos en Sistemas de Potencia) desarrollada como trabajo de grado en el programa de Ingeniería Eléctrica [27]. La herramienta consta de tres módulos que son:

El módulo para análisis en la frecuencia, el módulo para análisis en el tiempo y el módulo con análisis híbrido, que integra los dos algoritmos para producir una solución más eficiente respecto a tiempo de simulación y precisión en los resultados, para sistemas lineales con cargas no lineales conectadas.

\section{Módulo de frecuencia}

En el módulo de frecuencia el sistema es construido utilizando un menú gráfico para seleccionar los modelos. En la Figura 11 se identifican varios de sus principales elementos e íconos. En este módulo se calcula la tensión en los nodos del sistema, los datos son presentados en forma gráfica para cada nodo y en forma numérica en la ventana de comandos de MATLAB. La herramienta permite diferenciar los nodos del sistema mediante un código de colores aleatorio. A mayor número de elementos y nodos, el patrón de colores puede repetirse creando una aparente ambigüedad en la identificación visual. Mediante la opción Ver Número de Nodo, se muestra en la ventana de comandos el nodo seleccionado (ver Figura 12).

Los resultados del programa son las tensiones en los nodos; para el cálculo de otras variables se requiere un postprocesamiento de estas tensiones. Para esto la herramienta brinda un ícono para exportar los datos en diferentes formatos. El destino y el nombre del archivo a exportar aparecen en la ventana de comandos.

A continuación se presentan los resultados de algunos ejemplos, empezando por una bancada trifásica Dy11, construida a partir del modelo del transformador monofásico. La conexión del sistema y las formas de onda de la simulación son mostradas en la Figura 13. En la Tabla 1 se 


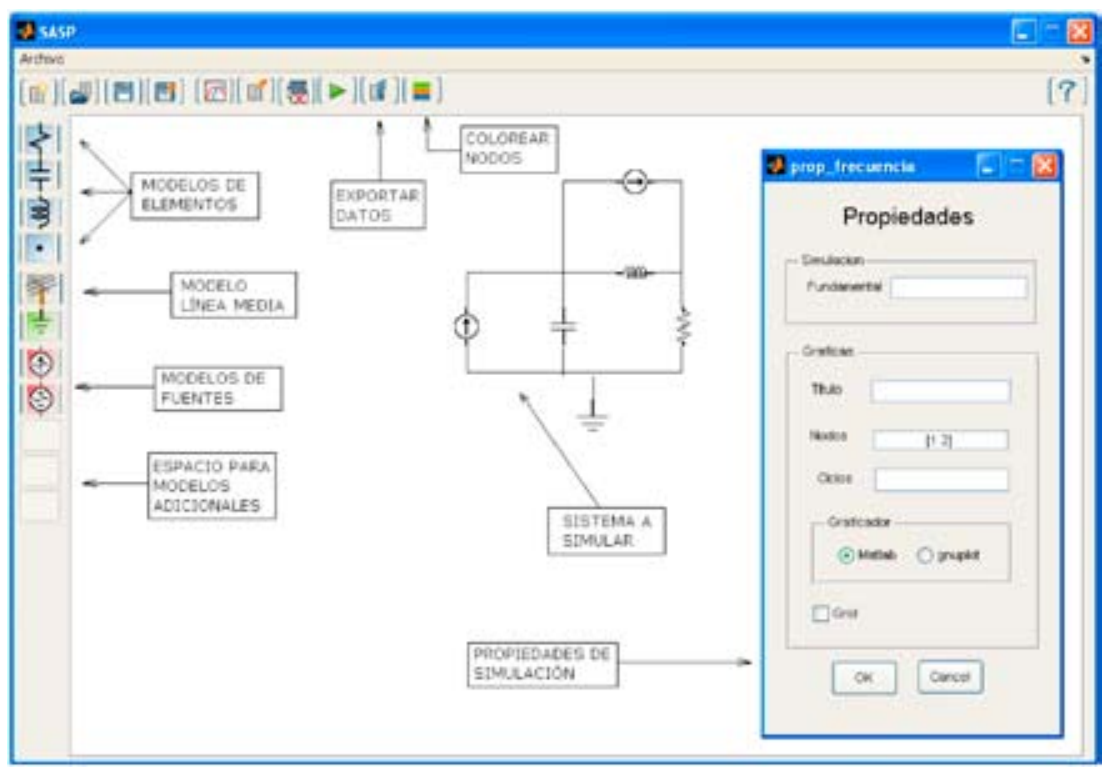

Figura 11. Módulo de frecuencia.

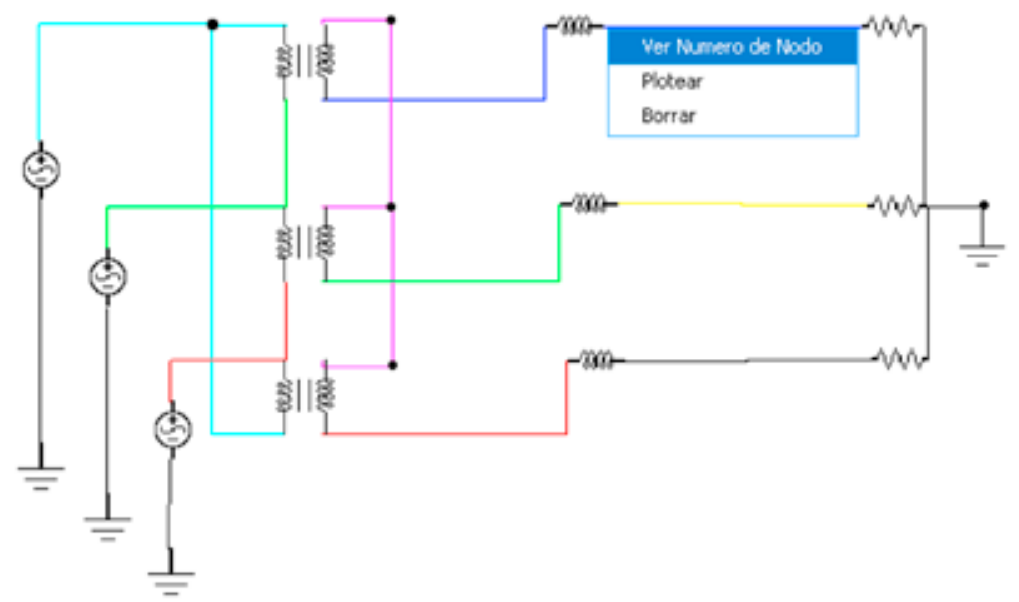

Figura 12. Codificación de colores redundante.

muestran los resultados de las tensiones en p.u. de los nodos de un transformador trifásico elevador en bancada Dy11.

Las tensiones de línea en el lado de la fuente y de la carga son calculadas a partir de la tensión en los nodos, de acuerdo con la expresión:

$$
\begin{aligned}
& V_{A B}=V_{4}-V_{2}=1,732 \angle 30^{0} \\
& V_{a b}=V_{8}-V_{9}=30,213 \angle 56,56^{0}
\end{aligned}
$$

Tabla 1. Tensión de nodo en la bancada trifásica Dy11.

\begin{tabular}{|c|c|c|}
\hline & \multicolumn{2}{|c|}{ Resultado SASP } \\
\hline Nodo & Magnitud & Ángulo \\
\hline 1 & 2,293 & $-56,19$ \\
\hline 2 & 1,000 & $-120,00$ \\
\hline 3 & 1,000 & 120,00 \\
\hline 4 & 1,000 & 0 \\
\hline 5 & 0 & 0 \\
\hline 6 & 2,294 & $-179,21$ \\
\hline 7 & 2,290 & 63,77 \\
\hline 8 & 17,437 & 26,25 \\
\hline 9 & 17,447 & 93,76 \\
\hline 10 & 17,437 & 146,22 \\
\hline
\end{tabular}




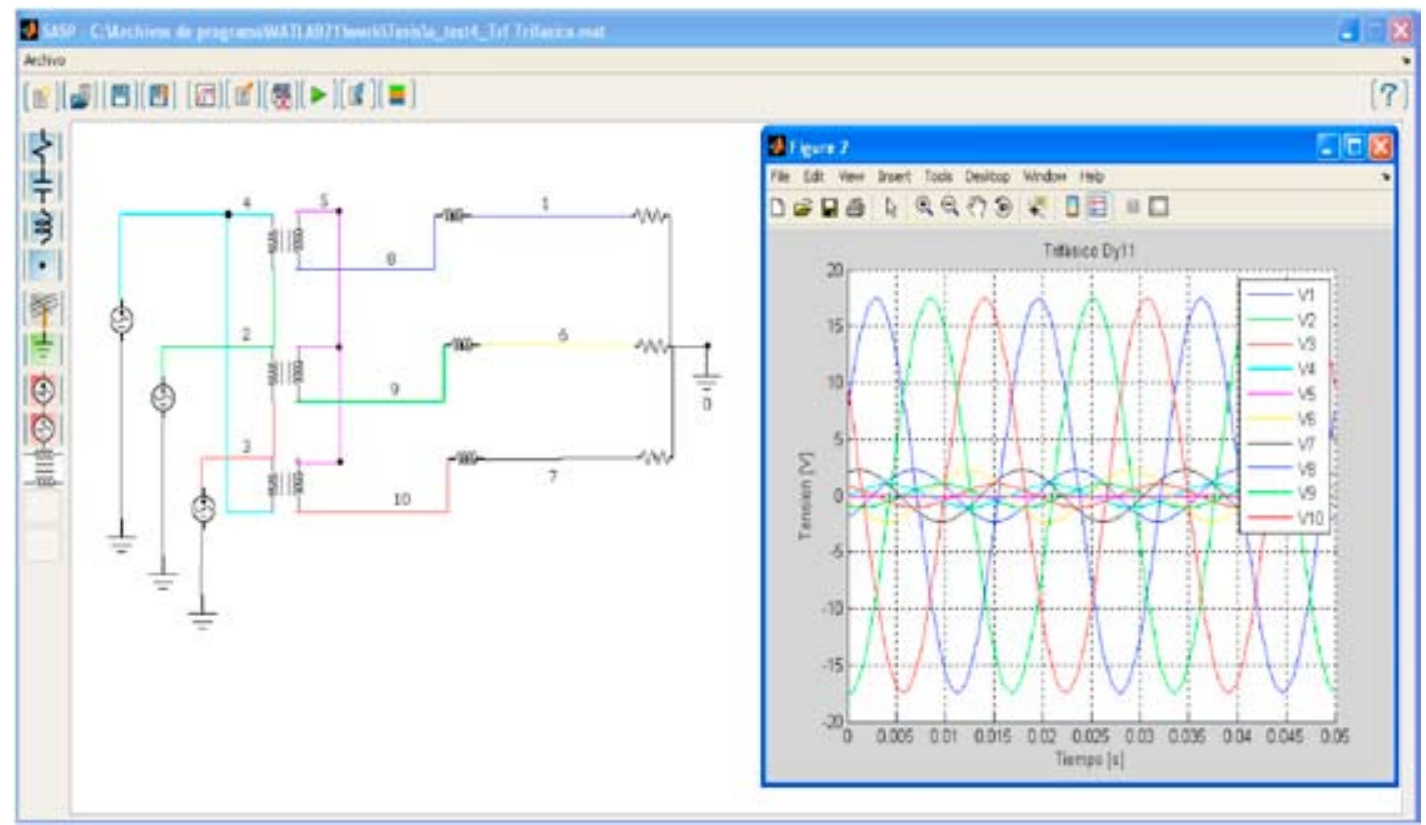

Figura 13. Bancada trifásica Dy11 con carga RL

\section{Módulo de tiempo}

El módulo de tiempo del programa SASP cuenta con un entorno similar al del módulo de frecuencia. Esta herramienta es apropiada para la simulación de circuitos de conmutación y otros elementos no lineales o variantes con el tiempo. El entorno gráfico permite incluir otros modelos, que deben ser creados en un archivo de texto a partir de las funciones primitivas y los modelos básicos de elementos.

Los tiempos de simulación dependen de las características del equipo de cómputo utilizado para las simulaciones. Este proyecto utilizó un equipo DELL, $1.66 \mathrm{GHz}, 1 \mathrm{~GB}$ RAM con MATLAB 7 SP3 y Windows XP Home Edition. Otros factores que determinan la duración de la simulación son: el intervalo y el paso de simulación, los cuales deben seleccionarse de forma tal que la resolución de la forma de onda permita diferenciar las distorsiones correspondientes a la respuesta no lineal del sistema de las distorsiones generadas por el paso de simulación seleccionado.

En la Figura 14 se muestra un rectificador trifásico de seis pulsos con carga RL y diodo de libre paso. El paso de iteración es de $6,2498 \times 10^{-5}$ segundos con un intervalo de simulación de 0,025061 segundos correspondiente a 1,5 ciclos de la fuente y 9 ciclos de la tensión rectificada en terminales de la carga.

En la Figura 15 se muestran la tensión en la carga (tensión entre nodos 4 y 5) y su corriente (traza roja).

La corriente se obtuvo por postprocesamiento y se escaló por un factor de 10 para hacerla comparable con la tensión y verificar los instantes de conmutación. El retardo en la activación de los tiristores corresponde a un disparo de $120^{\circ}$.

\section{Módulo híbrido}

Este módulo combina las ventajas de los métodos en el tiempo para simular circuitos no lineales o con elementos variantes en el tiempo, con las ventajas de los métodos en frecuencia para la simulación de la componente lineal de la red a estudiar. En el entorno gráfico se conectan subsistemas conformados por redes lineales y sistemas no lineales. Así cada porción del circuito queda en el dominio más eficiente (en cuanto a cantidad de cálculos y tiempo de ejecución) para su simulación.

El paso de simulación $(\Delta t)$ de los subsistemas en el dominio del tiempo es modificado de manera automática por el algoritmo cuando se calcula la 


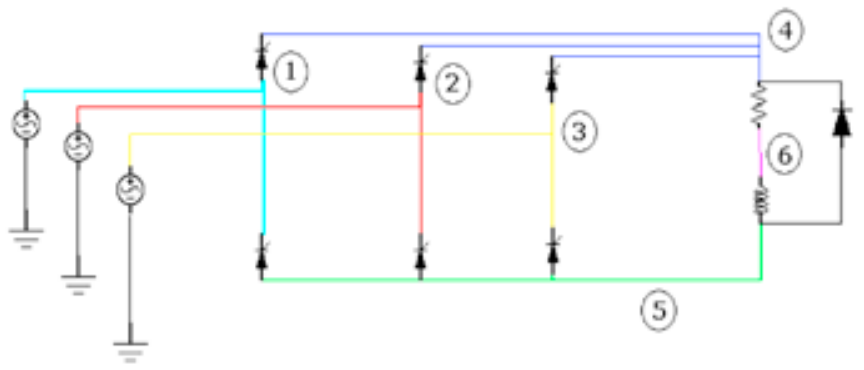

Figura 14. Rectificador trifásico controlado con diodo de libre paso.

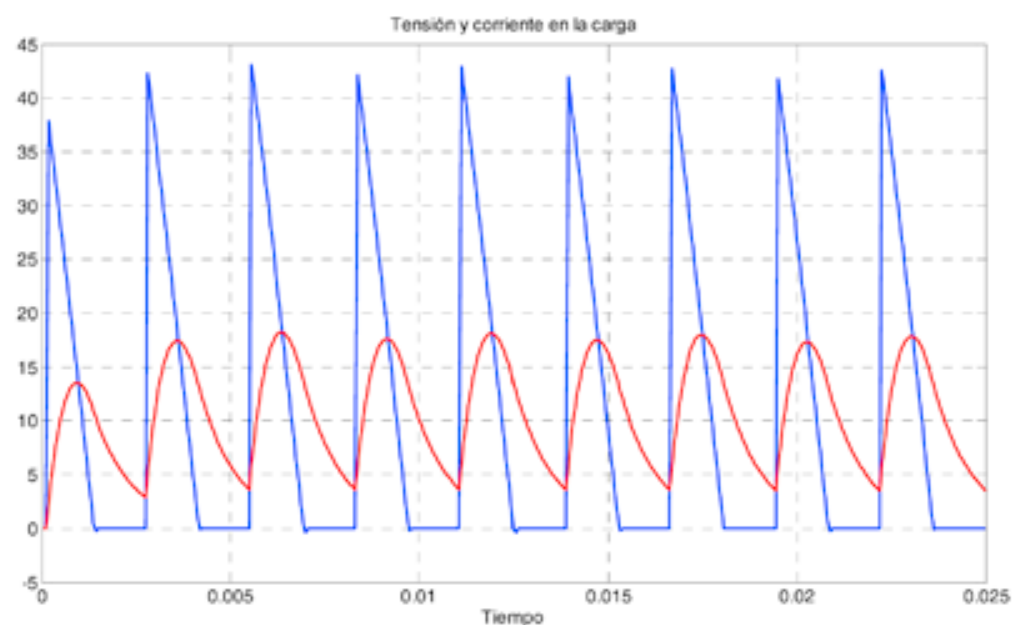

Figura 15. Formas de onda rectificador trifásico controlado con diodo de libre paso.

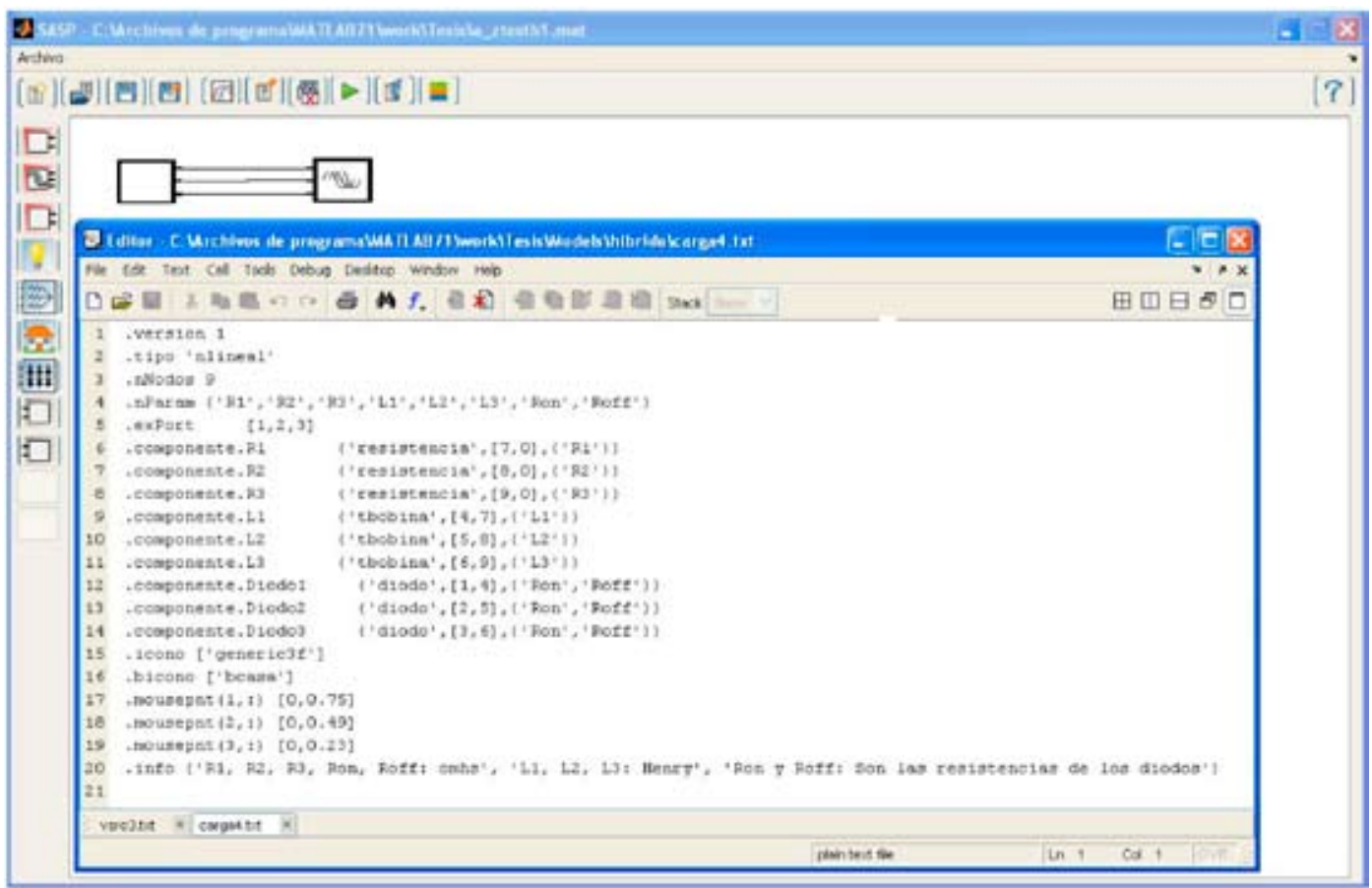

Figura 16. Entorno para el análisis del método híbrido. 
interacción entre las redes lineales y no lineales. En la Figura 16 se muestra el entorno para el análisis del método hibrido. Allí se observa una ventana con el modelo utilizado para la carga. El modelo debe ser previamente creado y puede ser incluido en el menú de elementos. Los bloques constitutivos de las simulaciones en el método híbrido corresponden a una biblioteca de modelos de cargas y redes lineales y no lineales previamente creados en modo texto.

Los resultados de la simulación son las tensiones en los nodos de cada uno de los sistemas lineales y no lineales, y la forma de onda y el espectro de la corriente entregada a cada una de las redes.

Para evaluar las corrientes, el algoritmo introduce de forma automática un ampermetro virtual en cada una de las líneas que conecta cada una de las subredes del sistema. El usuario puede seleccionar un valor adecuado para la impedancia de los ampermetros, dependiendo del valor de las impedancias relativas de los demás componentes del circuito para minimizar el efecto de carga. En la Figura 17 se muestra la posición de los ampermetros colocados de forma automática al interconectar las redes lineal y no lineal.

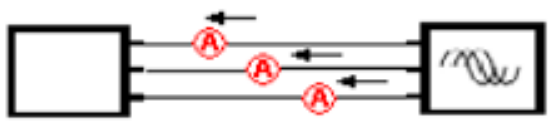

Figura 17. Ampermetros virtuales método híbrido.

En la Figura 18 se muestra el resultado para la tensión en los nodos del sistema no lineal. En las Figuras 19

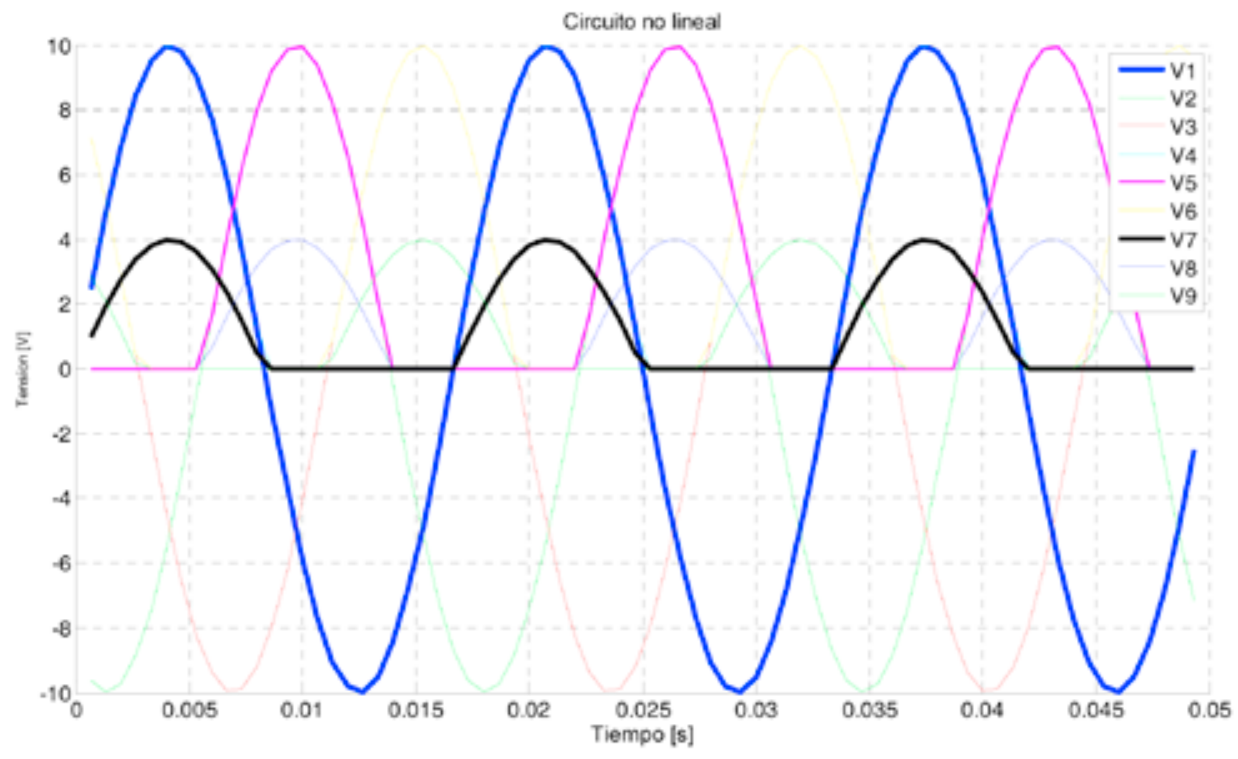

Figura 18. Tensión en los nodos de la carga no lineal.

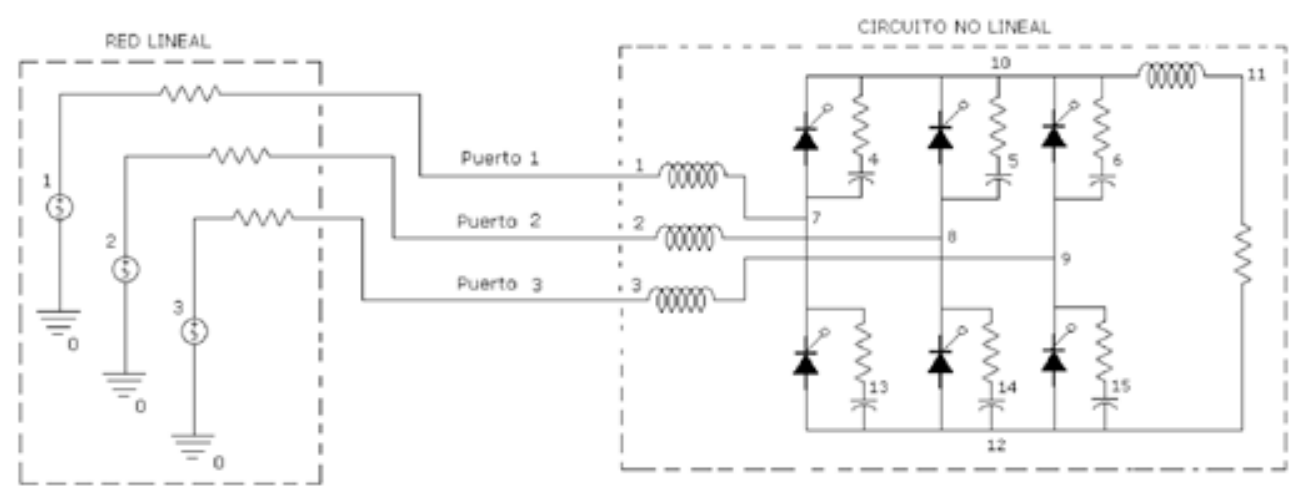

Figura 19. Circuito rectificador controlado. 


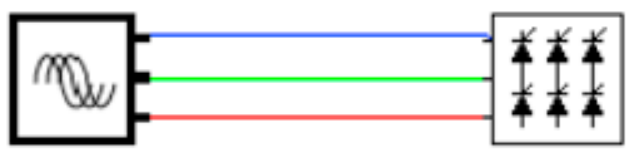

Figura 20. Rectificador controlado. Gráfica en SASP.

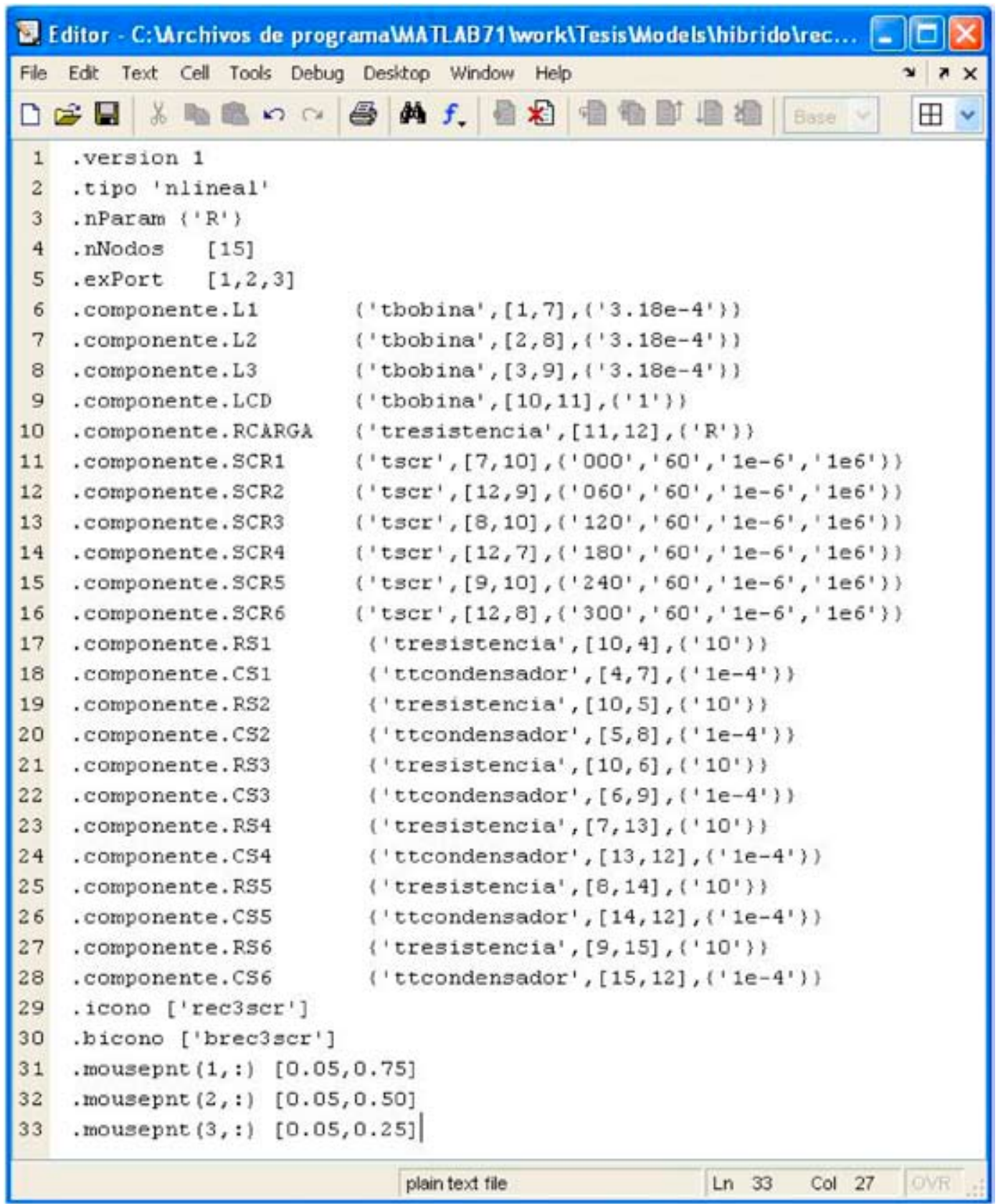

Figura 21. Rectificador controlado. Listado de red. 


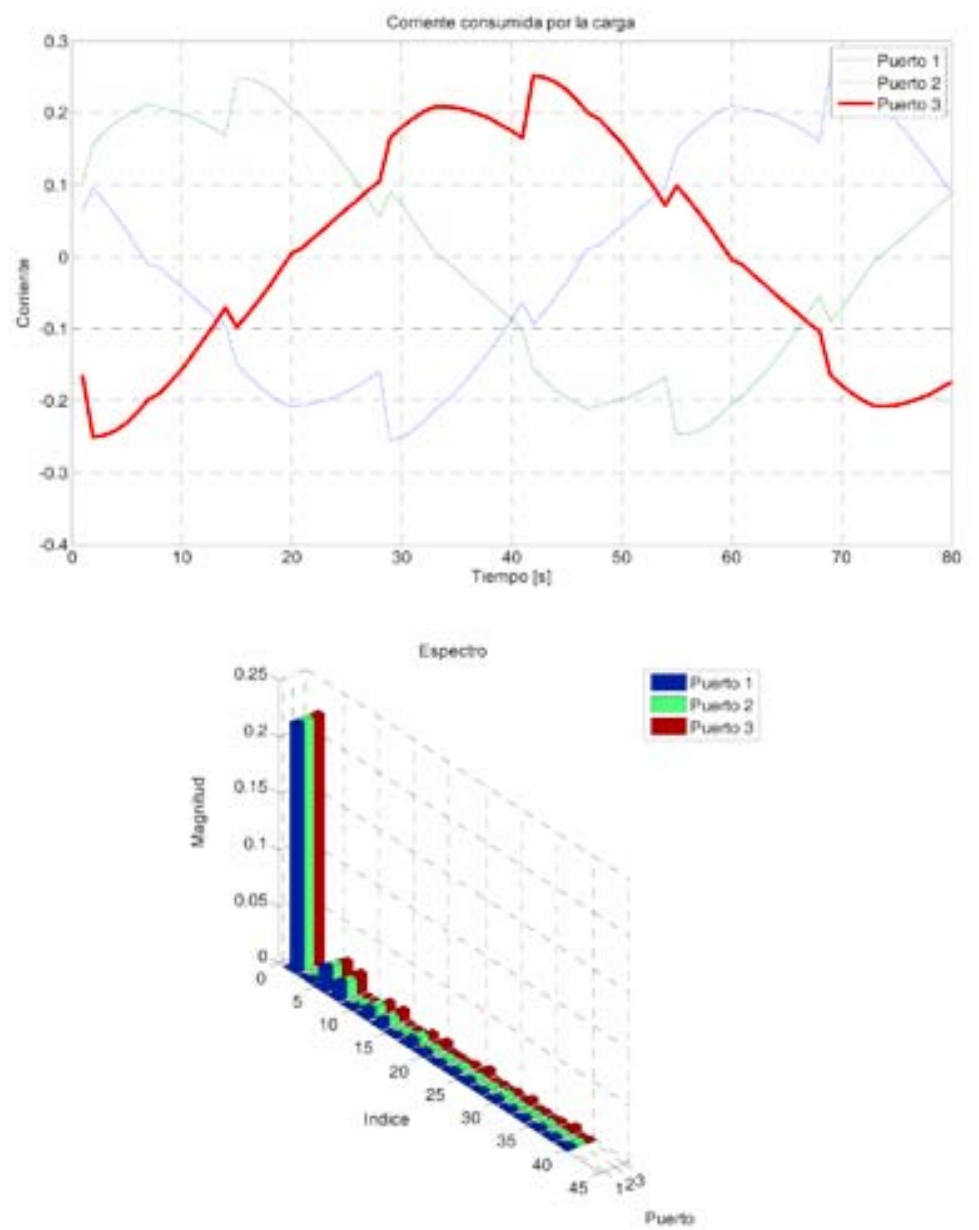

Figura 22. Forma de onda y espectro para las corrientes en el rectificador controlado.

a 21 se muestran: el esquema de un rectificador controlado, la conexión en el entorno híbrido del rectificador y el modelo de la carga no lineal creado como entrada de texto, respectivamente.

En las líneas 29 a 33 de la Figura 21 se crea el ícono para el espacio de trabajo (icono), el botón para el menú de elementos (bicono) y se dan las coordenadas (mousepnt) del punto de conexión correspondiente a cada uno de los nodos que van a interactuar con los demás subsistemas.

En la Figura 22 se observa la forma de onda de corriente suministrada a la carga. La simulación se realiza en dos iteraciones del método híbrido, con un paso final de 0,000208333 segundos un intervalo de simulación en estado estable de 0,03 segundos con 80 puntos por ciclo y un criterio de convergencia del $2 \%$ de la iteración anterior.

\section{CONCLUSIONES}

La precisión y convergencia en todos los circuitos y sistemas no puede ser garantizada por ningún método o simulador, ya que las condiciones particulares de cada circuito como la singularidad en la matriz de impedancias (corto circuito) o constantes de tiempo elevadas pueden causar que los resultados de la simulación diverjan.

La herramienta SASP muestra en cada uno de sus módulos las funciones básicas necesarias para generar matrices o vectores (cells) de resultados; de tal forma que con la información almacenada y utilizando postprocesamiento en la ventana de comandos pueden obtenerse otras variables del sistema.

La medida de tiempo como parámetro de eficiencia depende del tipo de procesador, memoria RAM, 
funciones básicas y llamados de interrupción del equipo utilizado en la simulación. Adicionalmente, también afectan los pasos e intervalos de simulación, número de puntos de la transformada de Fourier, cantidad de frecuencias armónicas a analizar, así como la exigencia en los criterios de convergencia. Las anteriores consideraciones permiten concluir que la medición de tiempos es relativa al tipo de computador así como a parámetros dependientes del usuario los cuales pueden cambiar entre una simulación y otra.

Se ha propuesto un método para la resolución de sistemas en los cuales se encuentran interconectadas cargas lineales y no lineales simultáneamente. La característica de las redes eléctricas es la presencia generalizada de elementos lineales con la aparición aislada de elementos no lineales concentrados en puntos específicos del sistema. Por lo tanto, resulta conveniente aprovechar la dimensión de los elementos lineales para simular esa porción del sistema en el dominio que reduzca su complejidad.

\section{AGRADECIMIENTOS}

Los autores expresan su agradecimiento a la Universidad Industrial de Santander, Bucaramanga, Colombia, por el soporte brindado para esta investigación.

\section{REFERENCIAS}

[1] J. Arrillaga, E. Acha and A. Semlyen "NewtonType Algorithms for the Harmonic Phasor Analysis of Non-linear Power Circuits in Periodical Steady State with Special Reference to Magnetic Non-linearities". IEEE Transactions on Power Delivery. Vol. 3, Issue 3, pp. 1090-1098. 1988.

[2] R.C. Dugan, M.F. Mcgranaghan, S. Santoso and H.W. Beaty. "Electrical Power Systems Quality". McGraw-Hill. Second Edition, p. 521. USA. 2004.

[3] A. Semlyen and M. Shlash. "Principles of Modular Harmonic Power Flow Methodology". IEE Proceedings - Generation, Transmission and Distribution, Vol. 147, Issue 1, pp. 1-6. January, 2000.

[4] A. Medina. "Harmonic Simulation Techniques (Methods \& Algorithms)". IEEE Power Engineering Society General Meeting. Vol. 2. July, 2003.
[5] "Modeling and simulation of the propagation of harmonics in electric power networks. I. Concepts, models, and simulation techniques". IEEE Transactions on Power Delivery. Vol. 11, Issue 1, pp. 452-465. January, 1996.

[6] R. Abu-Hashim, R. Burch, G. Chang, M. Grady, E. Gunther, M. Halpin, C. Harziadonin, Y. Liu, M. Marz, T. Ortmeyer, V. Rajagopalan, S. Ranade, P. Ribeiro, T. Sim and W. Xu. "Test Systems for Harmonic Modelling and Simulation". IEEE Transactions on Power Delivery. Vol. 14, Issue 2, pp. 579-587. April, 1999.

[7] S.J. Ranade and W. Xu. "An Overview of Harmonics Modeling and Simulation". IEEE Task Force on Harmonics Modeling and Simulation, p. 1. 2007.

[8] G.T. Heydt and D. Xia. "Harmonic Power Flow Studies: Part I and II". IEEE Transactions on Power Apparatus and Systems Vol. PAS-101, Issue 6, pp. 1257-1270. June, 1982.

[9] G.T. Heydt and J. Jun "Rapid Calculation of the Periodic Steady State for Electronically Switched, Time Varying Power Systems Loads". IEEE Transactions on Power Delivery. Vol. 11, Issue 4, pp. 1860-1867. October, 1996.

[10] J. Usaola. "Régimen Permanente de Sistemas Eléctricos de Potencia con Elementos no Lineales Mediante un Procedimiento Híbrido en los Dominios del Tiempo y de la Frecuencia", p. 81. Tesis para optar al grado de doctor. Director: Julio G. Mayordomo. Universidad Politécnica de Madrid. Madrid, España. 1990.

[11] M.A. Moreno. "Flujo de Cargas Trifásico Armónico Híbrido", p. 61. Tesis para optar al grado de doctor. Director: Julio Usaola. Universidad Carlos III de Madrid. Madrid, España. 2001.

[12] J. Contreras. "Régimen Permanente Rápido con Especificaciones de Potencia para el Análisis de la Distorsión Armónica en Redes Eléctricas”, p. 9. Tesis para optar al grado de doctor. Director: Julio Usaola. Universidad Carlos III de Madrid. Madrid, España. 1999.

[13] W.T. Wiechowski, J. Lykkegaard, C.L. Bak and B. Bak-Jensen. "Hybrid Time/ frequency Domain Modeling of Nonlinear Components". $9^{\text {th }}$ International Conference. 
Electric Power Quality and Utilisation. Barcelona, España. October, 2007.

[14] J.G. Mayordomo, M. Izzeddine, S. Martinez and R. Asensi. "A Contribution for Three Phase Power Flows using the Current Injection Method". Ninth International Conference on Harmonics and Quality of Power Proceedings, pp. 295-300. USA. 2000.

[15] G. Carpinelli, F. Gagliardi, M. Russo and D. Villaci. "Generalised Convertor Models for Iterative Harmonic Analysis in Power Systems". IEE Proceedings - Generation, Transmission and Distribution. Vol. 141, Issue 5, pp. 445-451. September, 1994.

[16] J.C. Das. "Power System Analysis. Short Circuit Load Flow and Harmonics". Marcel Dekker, Inc. USA. 2002.

[17] A. Shaban. "A MATLAB/SIMULINK Based Tool for Power Electronic Circuits". Proceedings of World Academy of Science Engineering and Technology. Vol. 37, pp. 274279. January, 2009.

[18] J.R. Martí and J. Lin "Implementation of the CDA procedure in the EMTP". IEEE Transactions on Power Systems. Vol. 5, Issue 2, pp. 394-402. May, 1990.

[19] Electronics Modelling Task Force \& Digital Simulation Working Group. "Guidelines for Modelling Power Electronics in Electric Power Engineering Applications". IEEE Transactions on Power Delivery. Vol. 12, Issue 1. January, 1997.

[20] E. Thunberg and L. Soder. "On the Performance of a Distribution Network Harmonic Norton Model". Ninth International Conference on Harmonics and Quality of Power Proceedings, pp. 932-937. USA. 2000.
[21] IEEE Std. 519-1992. "IEEE Recommended Practices and Requirements for Harmonic Control in Electrical Power Systems". 1993.

[22] J. Usaola and J.G. Mayordomo "Fast Steady State Technique for Harmonic Analysis". IEEE Transactions on Power Delivery. Vol. 6, Issue 4, pp. 1789-1790. October, 1991.

[23] E.B. Makram and D.P. Manjure. "Drawbacks of Linearization in Harmonic Analysis and Modeling". Ninth International Conference on Harmonics and Quality of Power Proceedings, pp. 926-931. USA. 2000.

[24] A. Semlyen and A. Medina. "Computation of the Periodic Steady State in Systems with Non-linear Components Using a Hybrid Time and Frequency Domain Methodology". IEEE Transactions on Power Systems. Vol. 10, Issue 3, pp. 1498-1504. 1995.

[25] P.D. Lieven Degroote, R.R. Wouter, R. Bert and V. Lieven. "Harmonic Analysis of Distribution Networks Including Nonlinear Loads and a Nonlinear Transformer Model". $3^{\text {rd }}$ IEEE Benelux Young Researches Symposium in Electrical Power Engineering. April, 2006.

[26] B.K. Perkins, J.R. Martí and H.W. Dommel. "Nonlinear Elements in the EMTP: SteadyState Initialization". IEEE Transactions on Power Systems Vol. 10, Issue 2, pp. 593-601. May, 1995.

[27] A.L. Moreno. "Desarrollo de una Interfaz Gráfica para la Solución de Flujos de Carga con Armónicos", p. 23. Trabajo de grado Ingeniería Eléctrica UIS. Director: César Antonio Duarte Gualdrón. Universidad Industrial de Santander. Colombia. Diciembre 2007. 